\title{
COMPUlSIVE HOARDING IN AN OLDER AdULT WITH AgGRESSION, DELUSIONS AND MEMORY LOSS: A Multimodality NeUROIMAgING Study
}

\author{
Vijay N. Chennamchetty ${ }^{1,2}$, Teresa J. Poprawski ${ }^{1,2}$, John W. Crayton ${ }^{1,2}$, Elizabeth A. Hamilton ${ }^{3}$, \\ Lukasz M. Konopka ${ }^{* 1,2,3}$ \\ ${ }^{1}$ Clinical Neuroscience Section, Hines VA Hospital Hines Il 60141, USA \\ ${ }^{2}$ Department of Psychiatry, Loyola University Medical Center, Maywood Il, USA \\ ${ }^{3}$ The Chicago School of Professional Psychology, 325 N Wells Str. Chicago Il 60610 Department of Experimental Medicine, USA \\ Received March 20, 2009; accepted March 29, 2009
}

\begin{abstract}
Multimodal assessment practices offer enriching contributions to linkages between clinical presentation and brain presentation. The following case study is an illustration of the effectiveness of structural and functional imaging in the diagnosis process. In this article a clinical vignette is given in which the client (Mr. B) presents with aggressive behavior, delusions, memory complaints and hoarding behaviors. The findings of the case of presented based on multimodal structural and functional imaging. Mr. B was evaluated using MRI (magnetic resonance imaging), EEG (electroencephalography), qEEG (quantitative EEG), LORETA (low resolution electromagnetic tomography), SPECT (single photon emission computed tomography), quantitative SPECT and neuropsychological testing.

Visual analysis of EEG recording revealed sharp waves with reversals primarily in the mid-central region $(\mathrm{Cz})$. Quantitative analysis of background activity acquired in eyes closed and alert state revealed increased absolute and relative beta power maximally distributed to the mid-central region $(\mathrm{Cz})$. This was consistent with the source analysis obtained from LORETA. Visual inspection of SPECT data indicated increased blood perfusion in the posterior cingulate cortex. The quantitative analysis of SPECT images, on the contrary revealed decreased perfusion in both anterior and posterior cingulate cortex. Furthermore, increased perfusion in bilateral sensori-motor cortices (right more than left) and decreased perfusion in the left posterior temporal lobe was observed. T-1 based MRI of the brain showed generalized cerebral atrophy. On neuropsychological testing Mr. B was able to complete only tests of lesser complexity. Base on published data Mr. B's structural and functional imaging can be linked to his clinical presentation and a potential diagnosis of Frontal Temporal lobe Disorder (FTD). The use of multimodalities and objective analysis of data can lead to more accurate case conceptualizations and linkages between brain structure/functionality and clinical presentation.
\end{abstract}

Key words: EEG; Hoarding Behavior; LORETA; Quantitative Neuroimaging; SPECT

\section{INTRODUCTION}

This is the case of Mr. B who was evaluated and provided psychiatric care during his inpatient stay at Hines VA Hospital in February 2004. The case was revisited three years later by one of the original team members to find answers to some questions that were left unanswered. Analysis of data in an objective fashion revealed unexpected and interesting findings.

Mr. B, an 83-year-old Caucasian male was brought to the emergency room from an assisted living facility because of uncontrollable aggressive behavior towards his wife and others. He had delusions (jealous type) that his wife was being unfaithful to him, and had made verbally aggressive threats to her in the past. He also made inappropriate sexual remarks to other residents of the facility. Talking with Mr. B's family revealed that he spent hours clipping articles from the newspapers, which he would then store all over his apartment. They reported that there was so much clutter in the apartment that it was difficult for one to move around, and that they had recently "thrown out tons" of newspaper clippings. Mr. B attempted to pursue accumulating newspa- 
per clippings even on the psychiatric inpatient unit. He demonstrated little insight into this behavior. He also had increasing forgetfulness and occasional nightmares about his experiences in World War II. At the time of admission his MMSE (Mini Mental Status Examination; Cockrell \& Folstein, 1988) score was 22/30 (impaired orientation to time and place, $0 / 3$ on 5 -minute recall, and impaired visuo-spatial skills). Mr. B was talkative, impulsive and grandiose at the time of presentation. He was given a working diagnosis by the primary team, of Bipolar I Disorder, most recent episode manic, with psychotic features (DSM-IV: 296.44) and probable AD (Dementia of the Alzheimer's type), with behavioral disturbance (294.11) (American Psychiatric Association, 2000). Multiple neuroimaging studies were conducted during inpatient stay to evaluate Mr. B's symptoms and behavior. An MRI of the brain (without contrast) was obtained to evaluate biological substrates that might explain his new onset agitation and delusions. The imaging showed an arachnoid cyst in the posterior fossa. Mr. B was seen by Neurology Service and the cyst was deemed not contributory to the clinical presentation. The lesion was deemed to be chronic in nature with no compression effects or associated neurological deficits. Neuropsychological evaluation was done upon recommendation of the Neurology service to evaluate dementia. Mr. B cooperated with basic neuropsychological testing (see methods section) but refused a more extensive battery of tests, preventing further neuropsychological evaluation. Brain SPECT and qEEG studies were also done to further evaluate potential functional abnormalities.

We hypothesized that a complex case such as this could be better conceptualized by objective analysis of data from multiple neuroimaging modalities.

\section{METHODS}

An MRI study was completed using a standard clinical protocol, eight days prior to performing EEG and SPECT. T1 and T2 sequences were used and a boardcertified (The American Board of Radiology) radiologist generated a clinical report.

EEG recording was done using 19 cephalic electrodes that were placed according to International 10/20 System. Electrode impedance was $<5 \mathrm{kOhm}$. The acquisition sampling rate was $500 \mathrm{~Hz}$, with filter setting at 0.1 $\mathrm{Hz}$ and $70 \mathrm{~Hz}$. Nose was used as a reference and ear lobe electrodes were active sites. The awake, eyes closed EEG was recorded. Post acquisition linked ears reference was computed. During the recording, $20 \mathrm{mCi}$ (740 MBq) of 99mTc-HMPAO (Hexamethyl Propylenamine Oxime) was administered via an established IV line from the outside of Faraday Chamber, for SPECT imaging. The patient was not aware of the injection time. The recording continued for an additional $10 \mathrm{mi}$ nutes following 99mTc-HMPAO administration.
A more detailed description of this procedure has been previously published (Goforth et al., 2004; Milo et al., ). The EEG recording was visually inspected to locate sharp waves and bipolar reversals. The quantitative analysis of background activity was performed using NeuroGuide 1.8.1 EEG software. The manually chosen, artifact-free epochs of 68 seconds duration were used for the analysis. The FFT (Fast Fourier Transform) was applied to assess the absolute and relative power at each frequency $(1 \mathrm{~Hz}-30 \mathrm{~Hz})$. NeuroGuide's normative database was used to evaluate the level of normality. The result of the statistical comparison was further evaluated by means of Key Institute's software LORETA, in order to define deviant sources of activity. Findings with standard deviation measures greater than \pm 2.0 were considered significant.

The SPECT data acquisition was performed approximately 90 minutes after ligand injection. A rotating gamma camera system equipped with ultra highresolution fan beam collimators (Trionix system) was used for SPECT data acquisition. Images were obtained in a $256 \times 128$ pixel format with an acquisition time of 50 seconds per step. Fan beam projection data were converted to parallel data in a $128 \times 128$ pixel format, and the SPECT reconstruction was performed with a Henning filter with 0.9 cycles/cm cut-off. Approximately 60 transverse slices were generated.4 A boardcertified (The American Board of Nuclear Medicine) nuclear medicine physician generated a clinical report.

The SPECT images were processed using Hermes software from HERMES medical solutions. The images were intensity-normalized and spatially co registered to the standard atlas. Statistical evaluation was obtained by means of BRASS software comparing the patient to the HERMES normative database using total counts adjustment. The abnormality threshold was set at 2.5 cubic $\mathrm{mm}$ and standard deviation of 2.5 (Radau et al., 2001).

Behavioral testing was also performed prior to acquisition of EEG and SPECT. Mr. B completed BDI (Beck Depression Inventory; Beck \& Steer, 1987) and BAI (Beck Anxiety Inventory; Beck et al., 1988). He attempted neuropsychological testing using CANTAB Expedio (Cambridge Neuropsychological Test Automated Battery), but could not finish the battery. He completed the simple tests successfully (MOT-Motor Screening, BLC-Big/Little Circle, first three stages of IED-Intra Extra Dimensional Set Shifting Task and SWM-Spatial Working Memory) but became frustrated and chose not to continue with the more complex last four stages of IED and SOC-Stockings of Cambridge. All psychotropic medications were discontinued prior to quantitative imaging studies. A urine drug screen done at the time of admission was negative for illicit substances. 


\section{RESULTS 1}

MRI: The images were read by a board-certified radiologist. The report listed the following findings. A 3.5 $\mathrm{cm}$ arachnoid cyst was seen in the posterior fossa, no mass effect was seen on the cerebellum or brainstem. Fourth ventricle appeared to be normal in size and location. In addition, a $6 \mathrm{~mm}$ low intensity lesion was seen in right tegmentum. This was noted to be either a cyst or an old infarct. Generalized cerebral atrophy with ventriculomegaly was also seen. Basilar and internal carotid arteries appeared to be patent, and no subdural collection or midline shift was noted (Fig. 1a, 2a).

EEG: Visual analysis of EEG record revealed sharp waves with reversals in the midline (primarily) and right hemisphere (Fig. 3a); Cz, C4, Pz, P4 regions. Strong driving patterns were noted during photic stimulation in addition to increased number of sharp waves and bipolar reversals in the central leads.

qEEG: The quantitative analysis of background activity showed statistically significant findings across all frequencies (delta, theta, alpha and beta), but mainly in beta frequency range $(12.5 \mathrm{~Hz}$ to $25 \mathrm{~Hz}$ ) [Table 1]. Excess absolute and relative beta power were observed in the midline. These findings were found to be present maximally at the vertex $(\mathrm{Cz})$ and at frequency of $14 \mathrm{~Hz}$ (absolute power, $\mathrm{z}$-value 3.99 ; relative power, $\mathrm{z}$-value 2.64). Other findings were excess higher frequency beta $(15 \mathrm{~Hz}-30 \mathrm{~Hz})$ absolute and relative power at $\mathrm{O} 2$ and T6.

LORETA: The midline findings of qEEG in the beta frequency were consistent with LORETA analysis. Once again, the abnormal activity was seen to be distributed in all frequencies (delta, theta, alpha and beta). The abnormalities were mostly seen in beta frequency and in the midline (Fig 2d). The maximum abnormality was seen at $14 \mathrm{~Hz}$ and was localized to cingulate gyrus (z-value 4.82). Abnormalities were also seen frontally in delta frequency, with maximal deviations from normal seen in the right middle frontal gyrus, $\mathrm{z}$-value 2.57 (Fig. 1d).

SPECT: Images were read by a board-certified nuclear medicine physician. The report indicated decreased perfusion in the bilateral anterior frontal lobes (Fig. 1b) and increased perfusion in the posterior cingulate gyrus (Fig. 2b). Findings consistent with prominent ventricles and a subarachnoid cyst in cerebellar midline were also noted.

qSPECT (quantitative SPECT): Statistical analysis of SPECT images showed decreased blood perfusion in the frontal lobes bilaterally (Fig. 1c), anterior and posterior cingulate gyrus (Fig. 2c), and posterior left temporal lobe. In addition, increased perfusion in bilateral sensori-motor cortices (right more than left) was seen (Fig. 3b).
Neuropsychological testing: Mr. B's scores on rating scales were very low for depression and anxiety (BDI: 6/63, BAI: 4/63). The symptoms endorsed were primarily somatic in nature. Mr. B only partially completed neuropsychological testing using CANTAB Expedio. He completed MOT (tests comprehension, learning and reversal) and BLC (tests for visual, movement and comprehension difficulties) sections successfully, indicating a capacity to understand and follow basic instructions (required for further testing using the CANTAB Expedio). The results of further testing are as follows. IED (tests rule acquisition and attention set shifting): $\mathrm{z}$ score -3.53 for the first three stages, $\mathrm{z}$-score -0.13 for errors (he was not able to complete the subsequent four stages). SWM (tests working memory and strategy use): z-score -3.61 for strategy, z-score -0.47 for errors. Mr. B did not complete SOC (tests spatial planning and motor control). He gave up after trying for about $10 \mathrm{~min}$ and $33 \mathrm{sec}$.

\section{RESULTS 2}

Mr. B presented with memory complaints, delusions and aggressive behavior. Based on his clinical presentation, he was initially diagnosed as having AD (Alzheimer's disease). His clinical picture of hypomanic symptoms also prompted his clinicians to make an additional diagnosis of bipolar disorder. Using only clinical presentation, absent neuroimages, the diagnosis was limited and incorrect. The compulsive hoarding behavior was left unaddressed in the context of the patient's more critical delusional and aggressive behavior. After obtaining and objectively analyzing results of neuropsychiatric imaging studies, it became clear that a diagnosis of FTD more closely fit the patient's condition. From this conclusion one could argue the importance of linkage between imaging and clinical presentation and also use Mr. B's neuroimaging information to represent FTD findings within the brain.

Other presentations that can be best understood using neuroimaging include Mr. B's disturbances of memory, a prominent feature of the patient's presentation. Decreased perfusion of left posterior temporal lobe as seen on quantitative analysis of SPECT might be reflective of hippocampal dysfunction, manifesting itself as memory impairment. Memory loss is a common feature of $\mathrm{AD}$, but is also prominent in various other types of dementias which can be distinguished from AD based on clinical neuropsychiatric features and neuroimaging. Individuals with FTD are more behaviorally disturbed and less depressed than those with $\mathrm{AD}$ (Levy, Miller, \& Cummings, 1996). In addition, delusions, agitation, elation/euphoria, disinhibition and irritability are seen more in persons with FTD as opposed to AD and other types of dementia (Rosen et al., 2006). Using previous research studies on neuroimaging one can make more 
efficient and direct empirical connections to clinical presentations and patient brain structures and functions. A recent study has shown a direct relationship between temporal hypoperfusion as seen on SPECT and hypomania-like behavior in FTD (McMurtray et al., 2006). Certain neuropsychiatric symptoms (euphoria, disinhibition, impulsivity and restlessness) of FTD can easily lead to an incorrect diagnosis of Bipolar Disorder, which is what occurred in the case of Mr. B. If head neuroimaging has been used initially in the diagnosis and treatment planning, the chances of effective practice would have been raised and mal practice would have been reduced.

Further imaging analysis would reveal structural and functional brain deficiencies that provide vital information to Mr. B's clinical behavioral presentations. Mr. B had difficulty with memory and the extent of his deficit was supported by his performance on MMSE and corroborated by his family members. He did not endorse symptoms of depression or anxiety at the time of presentation. MRI of the brain showed generalized cortical atrophy (Fig. 1a). Visual inspection of SPECT data suggested hypoperfusion in both frontal lobes (Fig. 1b) and this was confirmed with qSPECT (Fig. 1c). Quantitative EEG and LORETA showed increased delta power in the frontal lobes (Fig. 1d). These findings can explain Mr. B's impaired executive function as demonstrated on neuropsychological testing and inappropriate social behavior. In addition quantitative analysis of SPECT data confirmed a deficit in perfusion of the left temporal lobe. These findings suggest dementia, and again when the images are considered together, point to the diagnosis of FTD rather than AD (where the findings are more parieto-temporal). In addition, published data indicate that decreased perfusion of the left temporal lobe is associated with compulsive behaviors in FTD (McMurtray et al., 2006) Thus using multiple neuroimaging modalities resulted in a reevaluation of the clinical impressions which lead to better understanding of this case. Mr. B's case also pulls together empirically supported connections between neuroimaging and clinical presentation which offers more substantial support to the use of neuroimaging in case conceptualization.

Another set of interesting findings relates to $\mathrm{Mr}$. B's compulsive hoarding and his neuroassessment information. Though compulsive hoarding is seen in a wide range of psychiatric conditions (including dementia), it has traditionally been considered as part of OCD (Frost, Steketee, Williams, \& Warren, 2000). A large part of our understanding about compulsive hoarding behavior comes from studies of OCD patients using functional neuroimaging and neuropsychological testing. These studies suggest that dysfunction of the cingulate cortex may be responsible for hoarding behaviour (Devinsky, Morrell, \& Vogt, 1995; Awh, \& Gehring, 1999; Rauch et al., 2001).

The anterior cingulate cortex plays a role in motivation, executive function, focused attention, the assignment of emotional valence to stimuli, the monitoring of responses to conflict, emotional self control, problem solving, the detection of errors and of the selection of responses (Carter et al., 1998). The posterior cingulate cortex is involved in monitoring visual events, spatial orientation, episodic memory and processing of emotional stimuli (Vogt, Finch, \& Olson, 1992). PET (Positron Emission Tomography) studies have shown decreased metabolic activity in both anterior and posterior cingulate cortex, and increased metabolic activity in right sensori-motor cortex in compulsive hoarders (Saxena et al., 2004). Our qSPECT findings in the case of Mr. B replicate these findings (Fig 2c). An increasing number of studies are supporting the idea that compulsive hoarding is a neurobiologically distinct entity (Saxena, et al., 2004; Grisham, Brown, Liverant, \& Campbell-Sills, 2005). Our case points in that direction as well. Though Mr. B exhibited compulsive behavior, he did not meet the DSM-IV-TR criteria for OCD (obsessive compulsive disorder) (American Psychiatric Association, 2000) or OCPD (obsessive compulsive personality disorder) (American Psychiatric Association, 2000).

To further demonstrate the value of objective modalities, it is instructive to compare the results of the subjectively-interpreted SPECT with the objectively-analyzed qSPECT. The report of visual SPECT noted an increased perfusion of the posterior cingulate cortex (Fig. 2b). This finding is inconsistent with the available data indicating reduced cingulate metabolic activity in cases of compulsive hoarding (Saxena et al., 2004). However statistical analysis of quantitative SPECT data showed findings contrary to the ones seen on visual inspection. The initially-perceived increase in perfusion of posterior cingulate cortex was actually found to be a statistically significant deficit, when compared to a normative database (Fig. 2c). This is a clear reflection of the limitations of visual inspection in accurately localizing perfusion deficits or excesses. 
Table 1. Summary of MRI, EEG and SPECT results.

\begin{tabular}{|c|c|}
\hline Neuroimaging Study & Findings \\
\hline Brain MRI (Fig. 1a \& 2a) & $\begin{array}{l}\text { Generalized cerebral atrophy with ventriculomegaly. } \\
\text { A } 3.5 \mathrm{~cm} \text { arachnoid cyst in the posterior fossa with no mass effect on the cerebellum or brainstem. }\end{array}$ \\
\hline EEG (Fig. 3a) & $\begin{array}{l}\text { Sharp waves with bipolar reversals in the midline (primarily) and right hemisphere-Cz, C4, Pz \& } \\
\mathrm{P} 4 \text { regions. }\end{array}$ \\
\hline qEEG & $\begin{array}{l}\text { Statistically significant findings across all frequencies upon quantitative analysis of } \\
\text { background activity, but mainly in frequency range }(12.5 \mathrm{~Hz} \text { to } 25 \mathrm{~Hz}) \text {. } \\
\text { Excess absolute and relative power observed in the midline, present maximally at the vertex. } \\
(\mathrm{Cz}) \text { and at frequency of } 14 \mathrm{~Hz}(\text { absolute power, z-value } 3.99 \text {; relative power, } \mathrm{z} \text {-value } 2.64) \text {. } \\
\text { Excess higher frequency }(15 \mathrm{~Hz}-30 \mathrm{~Hz}) \text { absolute and relative power at } \mathrm{O} 2 \text { and T6. }\end{array}$ \\
\hline LORETA (Fig. 1d \& 2d) & $\begin{array}{l}\text { Abnormal activity seen in all frequencies. Maximum abnormality seen at } 14 \mathrm{~Hz} \text { and } \\
\text { was localized to cingulate gyrus (z-value } 4.82 \text { ). }\end{array}$ \\
\hline SPECT (Fig. 1b \& 2b) & Decreased perfusion in bilateral anterior frontal lobes. \\
\hline qSPECT (Fig. 1c, 2c \& 3b) & $\begin{array}{l}\text { Decreased perfusion in frontal lobes bilaterally, anterior and posterior cingulate gyrus and } \\
\text { posterior left temporal lobe. } \\
\text { Increased perfusion in bilateral sensori-motor cortices (right more than left) }\end{array}$ \\
\hline
\end{tabular}

Frequencies included: $\delta=1.0-3.5 \mathrm{~Hz}, \theta=4.0-7.5 \mathrm{~Hz}, \alpha=8.0-12.0 \mathrm{~Hz}$ and $\beta=12.5-25.0 \mathrm{~Hz}$

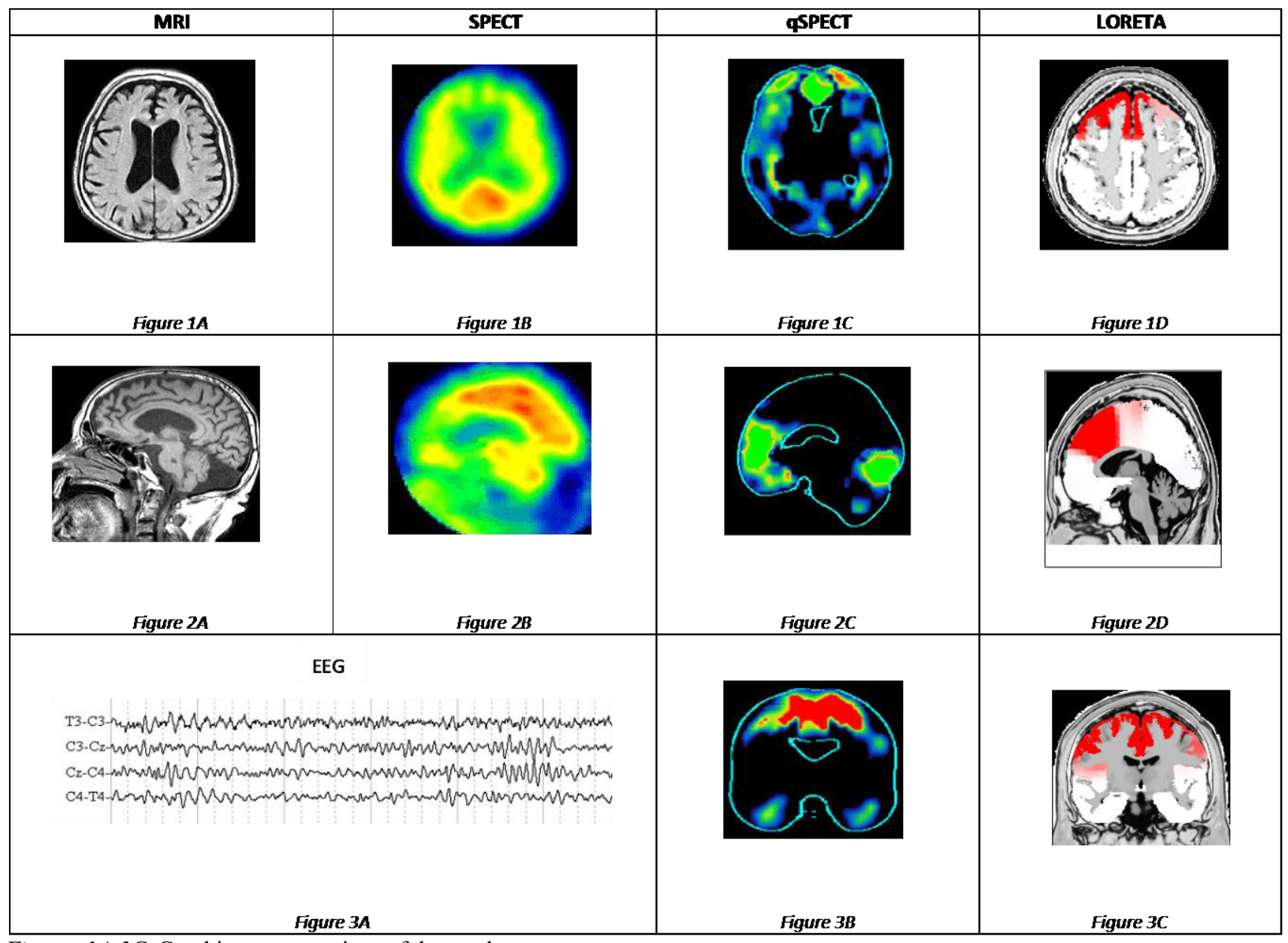

Figures $1 A-3 C$. Graphic representations of the results. 
The case of Mr. B demonstrates very well the convergence of results from behavioral testing and functional neuroimaging. Mr. B exhibited psychomotor agitation and endorsed several somatic symptoms on BDI and BAI. On visual inspection of the EEG (Fig. 3a) we saw sharp waves and bipolar reversals in the midline $(\mathrm{Cz}$, $\mathrm{Pz}$ ) and to the right of midline $(\mathrm{C} 4, \mathrm{P} 4)$. On qEEG we saw primarily an increase in absolute and relative beta power in the midline and regions adjacent to the midline, right greater than left (Fig. 3c). On qSPECT we saw increased perfusion in midline and bilateral sensorimotor cortices, right greater than left (Fig. 3b). We know that increased beta power is associated with increased metabolic activity as seen by PET (Oakes et al., 2004). Our case shows a correlation between increased beta power (qEEG) and increased perfusion (SPECT). The presence of sharp waves, increased beta power and increased perfusion in the sensori-motor cortices and it's relation to the presence of somatic complains by Mr. $\mathrm{B}$ brings the findings of multiple modalities together in a logical fashion to provide a more empirically and efficiently based form of diagnosis and treatment (Fig. 3a, $3 b, 3 c)$.

Neuroassessment has provided vital information to the diagnosis and treatment of a patient who might have otherwise received the incorrect case conceptualization resulting in an incorrect treatment plan. Through the case of Mr. B we would like to emphasize the importance of using multiple imaging modalities in evaluating complex behavioral presentations. The potential clinical benefits of this approach are a clearer understanding of the neurobiological substrate and better management of key presenting symptoms.

\section{Acknowledgement}

Financial support came from a VA Research Service Merit Grant to Dr. Lukasz Konopka.

\section{REFERENCES}

American Psychiatric Association (2000). Diagnostic and Statistical Manual of Mental Disorders. Fourth edition, Text Revision. Washington DC: American Psychiatric Press, 462463.

Awh, E., \& Gehring, W.J. (1999). The anterior cingulate lends a hand in response selection. Nature Neuroscience, 2, 853854.

Beck, A.T., Epstein, N., Brown, G., Steer, R.A. (1988). An inventory for measuring clinical anxiety: psychometric properties. Journal of Consulting and Clinical Psychology, 56, 893-897.

Beck, A.T., \& Steer, R.A. (1987). Beck Depression Inventory: Manual. San Antonio, TX: The Psychological Corporation.

Carter, C.S., Braver, T.S., Barch, D.M., Botvinik, M.M., Noll, D., Cohen, J. (1998). Anterior cingulate cortex, error detection, and online monitoring of performance. Science, 280, $747-749$.
Cockrell, J.R., \& Folstein, M.F. (1988). Mini Mental State Examination (MMSE). Psychopharmacology, 24, 689-692. Devinsky, O., Morrell, M.J., \& Vogt, B.A. (1995). Contributions of anterior cingulate cortex to behavior. Brain, 118, 279306.

Frost, R.O., Steketee, G., Williams, L., \& Warren, R. (2000). Mood, disability, and personality disorder symptoms in hoarding, obsessive-compulsive disorder, and control subjects. Behaviour Research and Therapy, 38, 1071-1082.

Goforth, H.W., Konopka, L., Primeau, M., Ruth, A., O'Donnell, K., Patel, R., Poprawski, T., Shirazi, P., \& Rao, M. (2004). Quantitative electroencephalography in frontotemporal dementia with methylphenidate response: a case study. Clinical EEG and Neuroscience, 35, 108-11.

Grisham, J.R., Brown, T.A., Liverant, G.I., \& Campbell-Sills, L. (2005). The distinctiveness of compulsive hoarding from obsessive-compulsive disorder. Journal of Anxiety Disorders, 19, 767-779.

Levy, M.L., Miller, B.L., Cumming, J.L., Fairbanks, R.A., \& Craig, A. (1996). Alzheimer disease and frontotemporal dementias. Behavioral distinctions. Archives of Neurology, 53, 687-690.

McMurtray, A.M., Chen, A.K., Shapira, J.S., Chow, T.W., Mishkin, F., Miller, B.L., \& Mendez, M.F. (2006). Variations in regional SPECT hypoperfusion and clinical features in frontotemporal dementia. Neurology, 66, 517-522.

Milo, T.J., Kaufman, G.E., Barnes, W.E., Konopka, L.M., Crayton, J.W., Ringelstein, J.G., \& Shirazi, P.H. (2001). Changes in regional cerebral blood flow after electroconvulsive therapy for depression. Journal of ECT, 17, 15-21.

Oakes, T.R., Pizzagalli, D.A., Hendrick, A.M., Horras, K.A., Larson, C.L., Abercrombie, H.C., Schaefer, S.M., Koger, J.V., \& Davidson, R.J. (2004). Functional coupling of simultaneous electrical and metabolic activity in the human brain. Human Brain Mapping, 21, 257-270.

Radau, P.E., Slomka, P.J., Julin, P., Svensson, L., \& Wahlund, L.O. (2001). Evaluation of linear registration algorithms for brain SPECT and the errors due to hypoperfusion lesions. Medical Physics, 28, 1660-1668.

Rosen, H.J., Allison, S.C., Ogar, J.M., Amici, S., Rose, K., Dronkers, N., Miller, B.L., \& Gorno-Tempini, M.L. (2006). Behavioral features in semantic dementia vs other forms of progressive aphasias. Neurology, 67, 1752-1756.

Rauch, S.L., Dougherty, D.D., Cosgrove, G.R., Cassem, E.H., Alpert, N.M., Price, B.H., Nierenberg, A.A., Mayberg, H.S., Baer, L., Jenike, M.A., \& Fischman, A.J. (2001). Cerebral metabolic correlates as potential predictors of response to anterior cingulotomy for obsessive-compulsive disorder. Biological Psychiatry, 50, 659-667.

Saxena, S., Brody, A.L., Maidment, K.M., Smith, E.C., Zohrabi, N., Katz, E., Baker, S.K., \& Baxter, L.R. Jr. (2004). Cerebral glucose metabolism in obsessive-compulsive hoarding. American Journal of Psychiatry, 161, 1038-1048.

Vogt, B.A., Finch, D.M., \& Olson, C.R. (1992). Functional heterogeneity in the cingulate cortex: the anterior executive and posterior evaluative regions. Cerebral Cortex 2, 435-443. 